\title{
Corela
}

Cognition, représentation, langage

HS-29 | 2019

Questions et exclamations au prisme de plusieurs approches linguistiques

\section{Les questions, wh et la thématisation, une enquête}

\section{Pierre COTTE}

\section{(2) OpenEdition}

Journals

Édition électronique

URL : https://journals.openedition.org/corela/8704

DOI : $10.4000 /$ corela.8704

ISSN : 1638-573X

Éditeur

Cercle linguistique du Centre et de l'Ouest - CerLICO

Référence électronique

Pierre COTTE, «Les questions, wh et la thématisation, une enquête », Corela [En ligne], HS-29 | 2019, mis en ligne le 06 septembre 2019, consulté le 21 septembre 2021. URL : http://

journals.openedition.org/corela/8704; DOI : https://doi.org/10.4000/corela.8704

Ce document a été généré automatiquement le 21 septembre 2021.

\section{(c) (i) (3) (2)}

Corela - cognition, représentation, langage est mis à disposition selon les termes de la licence Creative Commons Attribution - Pas d'Utilisation Commerciale - Partage dans les Mêmes Conditions 4.0 International. 


\title{
Les questions, wh et la thématisation, une enquête
}

\author{
Pierre COTTE
}

\section{Introduction}

1 J'appelle «thème » un terme support qui reçoit l'apport d'un "propos », qui lui est relatif. La relation thème-propos est générale et elle vaut à différentes échelles : textes, parties de texte, propositions, parties de proposition, ce qui est thème à un niveau pouvant être propos ou thème à un autre niveau. ${ }^{1}$ Une telle relation est construite. L'opération de thématisation choisit un terme librement, ou de façon contrainte dans ce qu'un texte offre ou impose, puis elle l'institue support pour un apport, et cadre d'une énonciation. Cette étude examine son rôle, souvent négligé, dans les questions, en particulier en wh.

\section{Rapport thème / propos dans les questions en wh}

\subsection{Exemple $1:$ le contexte}

2 Le chapitre d'où est extrait le texte à étudier relate les remémorations d'un personnage (Iris). Dans le premier énoncé So - on to the 'next thing, Madam' Iris s'encourage à se souvenir encore (en contexte the next thing signifie the next thing to remember). Cet énoncé annonce un thème au niveau du texte; le suivant The change in George! l'identifie en thématisant l'événement signifié par le syntagme nominal. L'exclamation exprime l'importance de ce thème: il est urgent de construire son propos; cette construction est le référent de it dans Iris couldn't put it off any longer. Le propos consiste en deux propositions interrogatives When had that begun? What was the cause of it? où that et it réfèrent à l'événement thématisé. Tout en constituant des propos, ces questions thématisent l'origine du changement, instituée sous-thème du texte, et elles appellent à leur tour le propos d'une réponse quant à cette origine. Le deuxième 
paragraphe décrit la difficulté de répondre ; l'interrogative de fin But when exactly had his abstraction become something more than natural ? précise When had that begun ? C'est à elle que s'enchaîne la réponse du début du troisième paragraphe; cette dernière est le deuxième propos du thème principal the change in George.

Le rapport entre question et thème explique qu'en anglais et en français le nom question puisse signifier « sujet d'actualité, problème dont on parle; a matter that needs to be dealt with or considered» et que les locutions in question/en question signifient "that I am discussing/talking about» (les dictionnaires) (sur la "question argumentative», cf. C. Plantin 2016: 492-6). Le couple question-réponse illustre parfaitement la distinction thème-propos, mais il est peu cité à cette rubrique : la propension à étudier les unités de la grammaire conduit à définir thème et propos comme parties de la proposition plutôt que d'une unité supérieure; en outre le travail de définition des linguistes s'appuie principalement sur les propositions déclaratives, jugées fondamentales. On propose ici que les interrogatives partielles sont thématisantes, dans la proposition et dans le texte.

\subsection{Le préconstruit}

4 L'interrogative But when exactly had his abstraction become something more than natural ? est un exemple de discours transposé, en l'espèce de discours indirect libre. Le past perfect y exprime la perspective du narrateur (cette forme dépend du temps de la narration); la syntaxe de discours direct exprime la perspective de celle qui s'interroge ; son choix traduit une empathie. Dans une telle interrogative la question porte sur une partie du procès. Le procès his abstraction had become something more than natural at some point est tenu pour vrai par l'énonciateur, ce qui autorise, dans le présent de l'énonciation, la question sur une de ses circonstances. Dans un autre texte avec la même interrogative, le procès pourrait être considéré vrai par l'allocutaire mais être jugé faux par l'énonciateur; la question, polémique, servirait à combattre la proposition, l'énonciateur mettant l'allocutaire au défi d'y répondre. Quel que soit le cas, la notion de "préconstruit » s'applique: dans l'interrogative analysée, le procès tenu pour vrai est préconstruit et l'énonciation construit la question.

5 L'interrogative est introduite par but. Cette conjonction signifie une opposition entre un premier, souvent concédé, et un second, auquel une force supérieure est donnée. À la ligne 6 elle opère une double opposition, rendue possible par la distinction, dans l'interrogative, du préconstruit et de la question. Il y a opposition: 1) dans le texte, entre le jugement That was all natural enough précédant l'interrogative et le jugement préconstruit contenu dans celle-ci (his abstraction had become something more than natural) la question n'entrant pas dans l'opposition ; 2) dans l'interrogative, entre le même jugement préconstruit et la question (when?). La première opposition est argumentative; but concède le jugement formulé en premier et accorde une force supérieure au second: la conclusion à en tirer (ce changement doit être expliqué) annule une conclusion inverse inférable du premier. Le linéaire reflète les étapes de cette opposition, le second jugement étant focalisé en fin de paragraphe. Cette opposition reflète la perspective du narrateur, qui pose dans son texte, et focalise à l'attention du narrataire, la proposition his abstraction had become something more than natural. La seconde opposition est psychologique et elle traduit la perspective d'Iris; but, qui n'est pas concessif ici, tient pour vraie la proposition juste citée et concentre 
l'attention sur la question, dont il indique l'importance. Le procès présupposé est construit le premier; la question qui s'y rapporte est seconde et au premier plan énonciatif; but focalise le mot interrogatif, qu'il introduit directement. Ici l'ordre linéaire ne reflète pas les étapes du sens; il le ferait s'il était His abstraction had become something more than natural at some point; but when exactly? Un tel ordre n'est toutefois pas retenu, la cohésion du texte demandant que la première opposition, argumentative, soit signifiée comme elle l'est.

6 L'adverbe when est modifié par exactly; le moment du procès appartenant à un passé délimité et connu, Iris veut le préciser. Une question partielle reflète donc le jugement qu'une détermination, même non nulle, est insuffisante; inversement, poser une déclarative implique qu'on juge suffisante une détermination positive, même si elle est faible (cf. at some point ci-dessus).

\section{Principes de la syntaxe génétique : une grammaire motivée}

7 La grammaire d'une interrogative partielle est différente de celle, canonique, d'une déclarative. Cette grammaire exprime-t-elle la volonté de savoir et la demande de façon seulement conventionnelle ou est-elle aussi motivée? Si elle l'est, les faits grammaticaux propres à l'interrogative sont les traces intelligentes de l'activité dont la question procède, qu'ils permettent de reconstituer. Je fais l'hypothèse de la motivation de la grammaire, des structures syntaxiques et partant des phrases interrogatives, et je propose une analyse de ces phrases dans le cadre guillaumien d'une syntaxe génétique.

8 G. Guillaume, le père de la psychosystématique du langage, a proposé seulement les linéaments d'une théorie syntaxique. De façon générale, le «mécanisme de l'incidence " régit les relations au sein des unités syntaxiques et y conditionne l'ordre des mots (1989: 123-6). Cet ordre, décrété « fait de syntaxe majeur », peut refléter aussi l'expressivité. Plus important pour cette étude, une distinction est faite entre « syntaxe en résultat » et « syntaxe en genèse » $(1973: 216-218)$ :

Dans le Cours de linguistique générale, Ferdinand de Saussure insiste sur le caractère linéaire de l'expression linguistique et il emploie pour le caractériser le terme de chaîne parlée. Il fait état aussi, et grand état, du terme de syntagme. Les termes en question, chaîne parlée et syntagme, se rapportent avec exactitude au discours parvenu à l'effet, c'est-à-dire au discours résultat. C'est linéairement qu'il se développe. Mais ce développement linéaire, qui est de la syntaxe en résultat, procède d'une syntaxe en genèse, qui n'est pas, elle, linéaire de la même manière et n'appartient pas en soi à la chaîne parlée, laquelle n'est que la fixation en résultat de ce qu'a produit une syntaxe sous-jacente génétique, dont la syntaxe en résultat représente une saisie par le travers.

La syntaxe génétique n'est pas horizontale comme celle de la chaîne parlée, mais verticale, et elle consiste en des opérations de pensée profonde dont l'axe de successivité n'est pas celui du déroulement du discours effectif mais celui du temps opératif porteur de la transition du fait de langue au fait de discours. (...)

Cette successivité verticale, dont la successivité horizontale, celle de la chaîne parlée, n'est que la saisie en résultat, [...] consiste partout à faire régresser le fait de discours primitif au-dessous de lui-même, (...) en vue d'obtenir un nouveau fait de discours différemment expressif (...).

De toutes ces opérations délicates appartenant à la syntaxe génétique, l'ordre des mots dans la chaîne parlée fixe le résultat; mais cet ordre des mots fixant résultat n'est pas autre chose que la projection à plat, sur un plan horizontal, d'opérations 
qui ont eu lieu sur une autre dimension, dans la profondeur de l'esprit, plus exactement dans le temps opératif porteur de l'intervalle et de la transition langue/ discours.

La question de l'ordre des mots est incontestablement dominante en syntaxe. Mais on la déforme obligatoirement si l'on veut expliquer à partir de lui-même, sans plus, cet ordre, lequel a sa source même, et sa raison d'être ce qu'il est, dans des opérations de pensée dont successivement il traduit non pas le développement, mais tout au contraire la clôture, l'arrêt, opérations auxquelles - en un mot - il met l'une après l'autre un terme. Dans la chaîne parlée, les opérations en question prennent rang l'une après l'autre d'une manière selon laquelle la moins éloignée dans la chaîne parlée est la dernière survenue dans l'esprit. (...)

L'ordre de rangement est l'inverse de l'ordre de production.

La syntaxe de résultat rendue par l'ordre des mots procède, en tout état de cause, d'une interception portée par le travers de la syntaxe génétique et mettant un terme à quelque partie de celle-ci. C'est dire que la syntaxe de résultat veut être expliquée par la syntaxe génétique dont elle n'est que l'aboutissant (...).

Commentant ces lignes, Boone \& Joly (2004 : 422-423) soulignent des similitudes avec la grammaire générative transformationnelle :

Cette syntaxe génétique «sous-jacente » évoque étrangement la syntaxe profonde des générativistes. Les similitudes sont évidentes : l'idée d'une suite d'opérations profondes (verticales) ordonnées et même, bien que le terme n'apparaisse pas, de «transformations ».» (...) «Il y a donc une phrase de départ postulée qui subit diverses transformations. »

On note aussi que la notion, aujourd'hui courante, de "préconstruit » s'applique à ce « fait de discours primitif » qui « régresse au-dessous de lui-même » pour qu'advienne un «nouveau fait de discours». Le préconstruit linguistique implique une différence temporelle entre les parties d'une structure et autorise d'envisager une construction génétique par étapes.

11 Les disciples de Guillaume ont poursuivi les recherches sur les incidences (ex : Valin 1981, Lowe 2007) mais ils ont moins repris l'idée d'une syntaxe en genèse, ni ne semblent avoir examiné de ce point de vue les propositions non nominalisées. Cela justifie l'analyse présentée, sachant cependant qu'il n'est plus possible aujourd'hui de soutenir que le fait majeur, en syntaxe, est le seul « ordre des mots ».

\section{Application des principes de la syntaxe génétique à l'étude de l'interrogative partielle}

\subsection{Genèse sémantique de l'interrogative partielle}

Sémantiquement l'interrogative partielle a deux parties: une proposition logique signifiant un procès et la question, qui concerne un participant, un circonstant ou une entité liée à un participant du procès (question en whose). ${ }^{2}$ La proposition logique (PL) répète une proposition du cotexte: A. She said something - B. What did she say? (PL : she said something); ou elle est impliquée salva veritate par une première proposition : A. She spoke at length - B. What did she say? (PL : she said something). Dans le texte 1.14 la PL est un jugement motivé par la situation décrite: He behaved like a man who has had a shock (PL : something was the matter); de même 1.26 (PL : something was in his mind) ; 1.37 c'est une croyance ou un savoir non-situationnel a priori : Rosemary's best women friends were $a$ or $b$ or $c$ (certaines personnes ayant été amies de Rosemary, elles sont 
nécessairement identifiables); 1.7 la PL his abstraction had become something more than natural at some point est une information nouvelle dans le récit, mais une chose sue d'Iris, qui explique sa question. Les propositions logiques qui sous-tendent les questions ont diverses origines. Quel que soit le cas, une condition des questions est qu'elles soient tenues pour vraies - par l'énonciateur en général ou par l'allocutaire dans certaines questions polémiques ${ }^{3}$; à ce titre elles peuvent montrer des indices d'existence, comme some, qui exprime une quantification existentielle, et leur forme est déclarative même si elles n'ont pas été assertées.

La question de l'interrogative partielle concerne une entité impliquée par la proposition logique; elle cherche à identifier ou à catégoriser cette entité. Autorisée par cette proposition parce que celle-ci est jugée vraie, elle opère dans son cadre et elle s'ajoute à elle. Dans la genèse de l'interrogative elle est seconde. Lorsqu'elle est introduite, la proposition logique, qui est conservée avec son mode déclaratif implicite, est "préconstruite», tandis qu'elle est dans le présent de l'énonciation, où elle promeut sa valeur illocutoire. Elle produit une nouvelle proposition, qui n'abolit pas, mais dépasse et intègre, la première, son soubassement. Cette nouvelle proposition est sans valeur de vérité ; ni vraie ni fausse, elle accomplit l'acte d'interroger, efficacement ou non.

14 Comment une proposition de type déclaratif est-elle transformée grammaticalement en proposition interrogative?

\subsection{Transformation grammaticale de la proposition logique en proposition interrogative}

On considère que la proposition logique sous l'interrogative 1.7 est His abstraction had become something more than natural at some point. Posant l'existence d'un procès et d'un seul, la proposition implique l'existence d'un moment et d'un seul avec lequel celui-là coïncide ; le syntagme prépositionnel at some point (in time) signifie la coïncidence avec ce «moment d'occurrence » appréhendé non comme durée mais comme situation dans le temps. Dans ce syntagme le nom point figure un moment sans étendue (un instant) et la préposition at est choisie en fonction de lui comme signe de coïncidence. Même si le SPrep at det. point peut renvoyer à un moment connu ou donné (at one point / at that point) une représentation abstraite convient à un moment que l'énonciateur conçoit mais ne connaît pas.

Le déterminant indéfini some signifie que le moment d'occurrence est une référence nouvelle dans le discours, à l'instar du procès. Il signifie aussi une quantité positive, synonyme d'existence. Existant, le moment d'occurrence unique correspond nécessairement à un et un seul élément déterminé d'un système indépendant de repères temporels, qui l'identifie - lui donne son identité « externe » (v. infra). Tout en le sachant, l'énonciateur ne peut pas établir cette correspondance; le moment du procès peut correspondre à une infinité de repères ; c'est une variable pouvant prendre alternativement telle ou telle valeur; cette ignorance de l'énonciateur est, avec la nouveauté et la positivité, un troisième trait du déterminant indéfini. ${ }^{4}$ Une indifférence à l'identité est possible (cf. some... or other) ; toutefois la tension entre la détermination certaine et l'ignorance comporte la possibilité d'une question visant à la résoudre. Conservant les traits de l'indéfini et cette tension, l'interrogative partielle demande qu'une unique valeur déterminée remplace la variable. Son principal trait grammatical est la présence de when au lieu du SPrep. D'autres traits grammaticaux sont l'avancée 
optionnelle de when et l'inversion Auxiliaire-Sujet corrélative dans une interrogative principale ou indépendante, ainsi qu'une réalisation phonétique focalisante.

\subsection{La thématisation comme opération première de la question partielle}

La thématisation est la première opération de la question partielle. Dans la proposition déclarative préconstruite une entité nominale est thème (ou thème principal) et le reste de la proposition est son propos. Typiquement ce thème déclaratif est le sujet et le propos est le "prédicat»; ex: His abstraction (thème) had become something more than natural at some point (propos). Cette relation thème-propos étant entérinée, la transformation interrogative thématise à nouveau, à l'intérieur de la proposition, ce dont elle demande l'identité : When (thème) had his abstraction become something more than natural ? L'entité thématisée est différente ici de celle qui l'est dans la déclarative, mais ce peut être la même (ex: Somebody called the police $\rightarrow$ Who called the police?). Énonciativement le thème interrogatif l'emporte sur le déclaratif: son propos, externe à la proposition, est la réponse attendue au premier plan énonciatif. Dans la déclarative préconstruite la relation thème-propos est complète; dans l'interrogative, elle est en construction. La proposition devient une partie d'une unité discursive supérieure, que la réponse du co-énonciateur doit compléter.

\subsubsection{Objets de l'opération de thématisation : identité externe et identité interne d'une entité nominale}

La thématisation de l'interrogative partielle concerne une entité nominale. Remplaçant le SPrep en at dans la proposition, when est un adverbe signifiant une coïncidence avec un moment. Cependant, la question à laquelle il contribue ne sélectionne pas la coïncidence, que l'énonciateur se donne, mais le seul moment signifié par some point dans la déclarative: les interrogatifs en wh des questions partielles thématisent une entité nominale. ${ }^{5}$

La thématisation interrogative concerne ensuite l'identité " externe ", déjà évoquée, de l'entité nominale, dont doit être distinguée l'identité «interne », aussi impliquée. a) En utilisant l'exemple du texte, l'identité interne de cette entité est «être le moment du procès signifié par la proposition particulière his abstraction had become something more than natural », formule où l'article défini signifie que ce moment est unique dans la catégorie des moments pour être celui de ce procès-là. Cette identité découle de la seule participation de l'entité au procès de la proposition - c'est pourquoi elle est « interne ». Elle est impliquée par la thématisation interrogative. Dans la déclarative initiale, le moment est quelconque; seul importe le fait qu'il existe comme site d'occurrence, et comme repère, du procès (d'où l'indéfini). Quand cette proposition est acceptée, le repérage, entériné, est inscrit en lui comme une propriété qui le distingue dans sa catégorie et qui constitue son identité interne. La question thématise le moment doté de cette identité ; elle suppose celle-ci car une question est viable seulement si les coénonciateurs s'entendent sur son objet. b) La thématisation interrogative vise l'identité « externe » du moment. Celle-ci est constituée par le repère temporel déterminé et indépendant auquel correspond nécessairement le moment du procès. Attribut a priori de tout moment qui existe, cette identité peut être ignorée; c'est elle que la thématisation interrogative sélectionne. 


\subsubsection{Variable, valeurs, parcours}

21 Dans la proposition sous-jacente et dans l'interrogative dérivée l'identité externe est une idée, une variable: un «terme indéterminé (...) susceptible d'être remplacé alternativement par divers termes déterminés (...), qui en sont les valeurs. " (Lalande 1926 : 1188). L'idée que les interrogatifs en wh signifient une variable a peut-être été introduite par 0 . Jespersen, qui parle de "unknown quantity $x$ » et de " $x$-questions" (1924: 303); elle est chez Lyons (1977: 757-8) et chez les auteurs comme Le Goffic (1994, 2002: 2007, 2015) dans une perspective culiolienne (Culioli 1990, 1999) ou Huddleston et Pullum chez les générativistes (2002: 35-6, 872 sq). Aux valeurs de la variable est souvent associée l'idée de parcours. Une formule comme « X est a ou b ou $\mathrm{c} »$ exprime un parcours. La conjonction de coordination maintient les valeurs séparées et égales comme valeurs possibles de la variable; elle les enchaîne en une séquence qui fait leur inventaire; le parcours est ici disjonctif : ou signifie que la sélection d'une valeur exclut la sélection des autres et qu'une seule valeur remplace la variable. ${ }^{6}$ Quand l'adverbe ever modifie un wh interrogatif il rend explicite un tel parcours, qualifié «sans issue » dans la Théorie des Opérations Énonciatives (TOPE) ; l'énoncé, expressif, connote la recherche et la difficulté de trouver la valeur déterminée qui remplace une variable.

(1) Whenever did you find time to do all that cooking?

De la proposition logique sous-jacente à l'interrogative dérivée le statut de la variable change. Dans la première proposition some pose l'existence d'un moment et du procès coïncidant avec lui : la proposition correspond à une situation; elle est jugée vraie et elle est de forme déclarative. L'identité externe du moment est une variable; cependant les valeurs ne sont pas parcourues: la quantification existentielle prime. Dans la deuxième proposition au contraire, la thématisation de when laisse la quantification existentielle dans le préconstruit et elle promeut l'identité externe inconnue, créant les conditions de la question ${ }^{7}$ : pour cette identité inconnue thématisée l'apport est la bonne valeur capable de la remplacer et l'énonciateur parcourt les valeurs.

23 La question est une suspension du mode déclaratif. À la première étape de la genèse, l'énonciateur a validé une proposition en some moins précise qui est entérinée ; à la deuxième étape, il veut valider une proposition plus précise où une valeur déterminée remplacerait la variable. L'échec de son parcours l'empêche d'asserter la proposition qui serait vraie, et la proposition en wh, sans valeur de vérité, est une proposition à mode déclaratif suspendu; la variable thématisée reste en l'état, ce qui motive l'invitation faite à l'allocutaire de fournir la bonne valeur. La réponse, qui produit une version enrichie, quoique parfois elliptique, de la proposition sous-jacente, rétablit le mode déclaratif.

\subsubsection{Rôle de wh interrogatif : critiques de la notion de parcours par la TOPE}

Le "parcours » a une place importante dans la Théorie des Opérations Énonciatives; c'est une "opération de détermination sur une classe, un ensemble ou un domaine notionnel consistant, pour l'énonciateur, à envisager successivement tous les éléments sans en choisir aucun (...)» (M.L. Groussier et C. Rivière 1996: 137). Certains énonciativistes pensent qu'il doit être précisé et critiquent, entre autres, l'étiquette 
« opérateur de parcours » appliquée à certains morphèmes, dont le wh interrogatif (cf. L. Gournay et G. Mélis 2006). Selon eux, le parcours tel qu'il est habituellement entendu est paradoxal : il explicite une indétermination pour « déterminer » une référence (L. Dufaye 2006); il est polymorphe, diffus à travers les marques (non territorialisé), intriqué avec l'extraction et le fléchage, voire implicite (G. Mélis 2006). Plutôt qu'une opération, c'est un complexe d'opérations, variable selon le type, si bien qu'un morphème dit «de parcours", par métonymie, peut ne signifier en propre qu'une partie de ce complexe.

Pour Mélis le parcours du wh interrogatif conçu comme traitement indifférencié, égalitaire d'unités distinctes est paradoxal en ce qu'il superpose différenciation et indifférenciation, pose les unités comme distinctes et indistinguables à la fois. Et ce paradoxe est coûteux. Certes les questions en which ou alternatives signifient un parcours; outre qu'il faudrait savoir quel protagoniste de l'énonciation est censé le faire, il est douteux qu'il ait lieu quand l'énonciateur ne dispose d'aucun objet identifié (à moins d'admettre un parcours d'objet non identifié à objet non identifié!) et si le sujet interrogé connaît la réponse, pourquoi envisagerait-il d'autres objets identifiés que le bon? Pour Dufaye, la question en wh parcourt bien des valeurs, mais le mot interrogatif signifie la cible, ou le site, du parcours, le paradigme des valeurs parcourues, non l'opération de parcours. Il en veut pour preuve l'analyse par Culioli (1985 : 72) de que dans Est-ce qu'il est venu? et le fonctionnement des phrases complexes à subordonnée interrogative; dans ces phrases, le verbe recteur (ask, wonder) signifie le parcours tandis que la tête de la subordonnée wh renvoie seulement à son objet. Gournay (2005) conteste pour sa part que wh renvoie véritablement à un paradigme de valeurs attendant sélection ou à un parcours : «Le fait qu'à la question « Who is coming ? » on puisse répondre Peter, John, Mary, My husband ne signifie pas que l'énonciateur construise cette diversité d'instanciables dans la question. La logique intuitive, qui nous permet d'envisager un balayage des valeurs, n'a pas à être prise en compte pour décrire une construction linguistique qui permet à l'énonciateur de poser l'existence d'une occurrence, ici d'animé humain, distinguée des autres par la propriété exprimée dans la relation prédicative " $\mathrm{X}$ is coming » (60). Dans ses différents emplois wh serait 1 ) une marque d'extraction signalant la (pré)construction d'une occurrence distinguée, déterminée qualitativement par la relation prédicative et dotée de "propriétés discriminantes même si celles-ci restent indéterminées»; 2) un relateur interpropositionnel: "l'élément «visé » par la valeur pronominale du marqueur se trouve repéré dans deux relations prédicatives, qui par son intermédiaire sont reliées entre elles» (63) (différence avec some). Pour R. Mauroy aussi $(2003,2006)$ les interrogatives en wh n'impliquent en elles-mêmes ni l'existence ni le parcours d'un domaine préconstruit d'occurrences repérées et définies, ces derniers s'ajoutant quand which est employé. Tous les wh marquent une "place vide " et, par là, un repérage interpropositionnel. Prenant en compte a) les propriétés qualitatives fournies par leur lexis, déterminée au plan du temps, de l'aspect et de la modalité, et b) le domaine ontologique signifié éventuellement par le mot interrogatif, les interrogatives en wh construisent un "domaine notionnel ouvert" pour la référence du mot en wh. Ce dernier renvoie à ce domaine et il implique la présence d'une "valeur qualitative différentielle » (occurrence particulière) en relevant, sans la signifier cependant (place vide). Le co-énonciateur répond dans le cadre ainsi posé. Pour l'auteur (Mauroy 2006), il n'est pas indispensable de présenter le terme désigné par wh ou la relation prédicative comme préconstruit(e) : «la lexis n'est pas d'emblée préconstruite comme assertée ou 
même validée sur les termes saturés (instanciés) » et some n'est pas le préconstruit de wh; il y a préconstruction toutefois dans les interrogatives clivées (ex: What is it that... ?).

\subsubsection{L'hypothèse génétique : wh interrogatif comme marqueur de parcours} questions en wh, quand l'énonciateur (au début d'une recherche) ne pense à aucune valeur particulière pour remplacer une variable. C'est ce qu'envisage Culioli (1985:70); à propos de la question Qui a touché à la crème ? il écrit : «j'ai alors parcours de tous les Qt1 : tel ou tel ou tel. 'Tel' ne renvoie à rien d'autre qu'une représentation par laquelle je désigne une valeur totalement abstraite. » Dans tel ou tel chaque tel pointe une entité située et distincte - ces qualités sont celles des valeurs et des repères - mais il ne l'identifie pas : le pointage et la valeur sont abstraits ; ils ne sont pas occurrences mais types. La conjonction de coordination garantit que chaque tel fait un pointage égal et différent; elle construit un parcours disjonctif abstrait. Ce qui est pointé ainsi n'étant pas identifié, un tel parcours ne peut rien sélectionner; en revanche, mettant le référent inconnu en relation tour à tour avec un nombre indéfini de valeurs, il montre l'incapacité à poser une correspondance stable avec une seule et il exprime, dynamiquement et indirectement, l'ignorance de l'énonciateur. L'hypothèse génétique de cet article propose que le some préconstruit implique un parcours abstrait repris par le when interrogatif.

3) Which n'a donc pas l'exclusivité du parcours, mais il est seul à signifier un parcours de valeurs identifiées. Il marque que la valeur cherchée appartient à un ensemble défini donné dans le contexte situationnel et/ou discursif et il renvoie par deixis ou anaphore à cet ensemble saillant, ce qui le dispense de signifier un domaine ontologique (temps, 
espace, personne, etc.). Les valeurs étant identifiées, une sélection est possible, mais elle échoue.

31 4) Qui fait le parcours? Lyons (755-7) distingue questionnement (wondering) et question (asking someone/oneself). Le premier est un acte mental: "It is one way of entertaining a proposition...in what we may refer to as the dubitative mode" ; éventuellement et à certaines conditions, il est transformé en acte illocutoire (question); dans le texte l'énoncé d'Iris est une question, au sens de Lyons. Or, questionnement et question ont la même grammaire et on peut penser que le parcours des valeurs est effectué en vain dans le questionnement de l'énonciateur, avant que la question n'invite l'allocutaire à l'entreprendre à son tour.

5) Le parcours interrogatif est un complexe d'opérations; le trajet sans sélection implique, entre autres, la construction d'une classe à parcourir et le pointage abstrait décrit ; il dépend aussi d'opérations énonciatives comme la thématisation. Outre que les opérations pertinentes mériteraient d'être recensées et articulées entre elles ${ }^{8}$, le problème se pose de leur expression par les marques grammaticales de l'interrogation, en particulier les mots en wh. De façon générale la correspondance entre opérations et marqueurs n'est pas biunivoque et elle est difficile à préciser ; le wh des interrogatives signifie-t-il le parcours, son objet, telle de ses opérations, les opérations qui l'encadrent ou autre chose ? Pour L. Dufaye (ou M.L. Groussier et C. Rivière), il construit seulement le paradigme des valeurs à parcourir, dans l'hypothèse d'une division des tâches entre des marqueurs dotés chacun d'un invariant minimal. Dans la théorie présentée ici, le wh interrogatif est riche en signification. Il est stratifié et opère sur un préconstruit complexe qu'il conserve: en résumé, la référence à une entité particulière qui appartient à un domaine ontologique et à la situation de la proposition (identité interne) et dont l'identité externe, inconnue, est une variable à la tête d'un paradigme de valeurs identifiées ou non. Son opération est la thématisation de cette variable; ainsi il met cette dernière en situation d'appeler et de recevoir l'apport de la valeur déterminée qui lui correspond et il déclenche le parcours des valeurs possibles. Il ne construit pas le paradigme des valeurs, tiré du préconstruit, et il signifie un complexe d'opérations parmi lesquelles le parcours des valeurs découle de l'opération principale de thématisation. Vraisemblablement les wh relatif et exclamatif sont analysables dans le même esprit. Maintenant si chaque marqueur peut signifier plusieurs opérations, une opération peut être signifiée par plusieurs marqueurs : le parcours des valeurs peut l'être par wh et par le signe spécialisé ever, ou par wh et un verbe comme ask ou wonder, associé à d'autres composants sémantiques! Pourquoi la construction de valeurs ne connaîtrait-elle pas la redondance ? L. Gournay reconnaît que le wh interrogatif renvoie à une entité indéfinie ayant implicitement une identité interne et externe. Cependant, faisant l'hypothèse d'un sens non stratifié et minimal, elle exclut que wh signifie aussi un parcours de valeurs. L'idée selon laquelle les opérations de la grammaire ne traduisent pas nécessairement une «logique intuitive» devrait être plus argumentée. Si la grammaire d'une proposition interrogative est seulement conventionnelle, il importe peu, à supposer que des opérations soient encore nécessaires, qu'elles soient telles ou telles et le parcours peut être négligé. Mais si on suppose que les marques grammaticales sont les traces d'une construction du sens, il est naturel d'envisager pour le wh interrogatif une opération de parcours, synonyme de recherche et de question. L. Gournay pense que wh a le même contenu minimal (opérateur d'extraction, relateur interpropositionnel) dans ses différents emplois; si c'est vrai, quel rôle a ce contenu, et quel est le rôle des autres marques grammaticales, dans la construction de 
la valeur interrogative? Comment celle-ci s'ajoute-t-elle à wh défini comme le veut Gournay? Dans le présent article, l'opérateur d'extraction est le some que wh remplace et conserve ; en thématisant une variable, ce dernier déclenche le parcours des valeurs et pose le cadre de la relation interpropositionnelle question-réponse. Enfin l'idée de Mauroy selon laquelle wh signifie une place vide à l'origine d'un repérage interpropositionnel est proche, à la thématisation près, de l'idée développée ici d'une variable promue thème pour un propos attendu. Pour Mauroy il n'y a parcours que d'occurrences repérées et définies, les wh interrogatifs, sauf which, ne renvoient ni à des occurrences ni à leur parcours et cela empêche some, marque d'occurrence, d'être leur préconstruit. Or les valeurs de certains parcours sont non identifiées (cf. some...or other, tel ou tel) et les wh interrogatifs expriment vraisemblablement de tels parcours; l'emploi cité de some montre que ce déterminant peut être leur préconstruit.

\subsection{La focalisation comme seconde opération de la question partielle}

La seconde opération de la question partielle est la focalisation. Grâce à l'accentuation et à l'intonation principalement, cette opération énonciative met en relief un segment d'énoncé (focus) et attire l'attention de l'allocutaire sur sa référence et éventuellement sur celle du matériau lexical voisin (focus large). Selon le mode, le focus est ce que l'énonciateur veut faire connaître (énoncé déclaratif) ou veut connaître (énoncé interrogatif): une question partielle focalise une identité externe (ou une catégorie d'appartenance) ignorée pour que l'allocutaire l'identifie; dans l'exemple du texte elle focalise le syntagme when exactly, où when prend l'accent nucléaire au sommet d'une courbe intonative descendante.

34 Typiquement, dans une proposition déclarative, thème et focus sont différents. En (3), le sujet his abstraction, qui est donné, est thème et le prédicat, qui porte un propos nouveau, contient le focus :

(3) But his abstraction had become something more than natural (at some point).

Mais dans une interrogative en wh, où aucune référence n'est nécessairement nouvelle, la thématisation et la focalisation promeuvent le même objet au premier plan énonciatif. Malgré cela elles sont distinctes. La première institue l'identité externe du moment du procès thème (support en devenir) pour un propos absent appelé; la seconde focalise cette identité externe thématisée pour attirer l'attention de l'allocutaire interrogé sur le fait qu'elle est thématisée et qu'elle attend un propos. Thématisation et focalisation se séparent à nouveau dans la réponse; celle-ci déclare et focalise une information nouvelle, le propos demandé ; elle peut se réduire à ce propos, dont le thème est dans la question.

Ce qui précède ne signifie pas qu'une interrogative en wh a une seule focalisation. Dans When exactly had his abstraction become something more than natural? l'information à partir de become est nouvelle et pertinente pour le récit ; elle bénéficie peut-être d'une focalisation secondaire en fin d'énoncé, mais l'interrogative focalise principalement when. La thématisation et la focalisation sont donc des conditions énonciatives du parcours interrogatif ; les mots interrogatifs en wh réunissent les trois opérations. 


\subsection{La thématisation comme motivation du mouvement de wh interrogatif}

\subsubsection{Précisions sur le rôle de thème de wh interrogatif} position. Les wh interrogatifs recherchent cette position parce qu'ils sont thèmes :

"The natural theme of a question, therefore, is 'What I want to know.' (...) In a whinterrogative, which is a search for a missing piece of information, the element that functions as Theme is the element that requests this information, namely the whelement. It is the wh-element that expresses the nature of the missing piece: who, what, when, how, etc. So in a wh-interrogative the wh-element is put first no matter what other function it has in the mood structure of the clause, whether Subject, Adjunct or Complement. The meaning is 'I want you to tell me the person, thing, time, manner, etc.'

Interrogative clauses, therefore, embody the thematic principle in their structural makeup. It is characteristic of an interrogative clause in English that one particular element comes first ; and the reason for this is that that element, owing to the very nature of a question, has the status of a Theme. The speaker is not making an instantial choice to put this element first; its occurrence in first position is the regular pattern by which the interrogative is expressed. It has become part of the system of the language, and the explanation for this lies in the thematic significance that is attached to first position in the English clause. Interrogatives express questions ; the natural theme of a question is 'I want to be told something' ; the answer required is either a piece of information or an indication of polarity" (75).

On peut penser avec ces auteurs que les mots en wh sont premiers parce que leur référent est thème ; mais des différences existent avec l'analyse du présent article :

1) la grammaire systémique fonctionnelle considère que la fonction thème est inhérente aux questions et à la première position, mais elle n'est pas génétique et ne connaît pas les mouvements : elle n'a pas de statut pour les relatifs in situ. Dans la présente analyse, le relatif est d'abord in situ. Il est en première position parce qu'il a la fonction sujet, thématisante aussi, ou parce qu'une avancée explicitante rend manifeste/exprime sa thématisation. Cela étant, dans les deux approches, le fait que wh, sujet ou non, soit le premier constituant référentiel de l'énoncé interrogatif est pertinent; il est motivé par le caractère thématisant du mot interrogatif et de la première position, wh choisissant une position affine éventuellement au prix d'un mouvement. En grammaire générative, où une interprétation pragmatique de la syntaxe est exclue, ce fait n'est pas pertinent. Les wh sujets et non sujets n'ont simplement pas la même position structurale dans la proposition, les seconds étant en position "prénucléaire " (avant le sujet et le prédicat) sans que leur avancée dans la structure soit expliquée.

41 2) Pour la grammaire systémique fonctionnelle, le thème est cadre plutôt que support ("[it] sets the scene for the clause itself and it positions it in relation to the unfolding text" 
(Halliday et Matthiessen 2004: 66)) et le "rhème " « développe " le thème plutôt qu'il n'est un apport. Par ailleurs le thème d'une proposition a un seul rhème, qui est dans la même proposition. Contrairement à l'intuition, un wh interrogatif est donc sans rapport avec la réponse, qui n'a pas de place dans la théorie au chapitre "message ». Dans l'analyse de cet article, la relation pertinente est support-apport. Dans une proposition déclarative où le thème est le sujet, le rhème (le prédicat) qui donne une information nouvelle, est seul apport; en revanche, dans une interrogative où wh est thème, deux apports existent. Le rhème (reste de la proposition) renvoie au procès présupposé de la proposition préconstruite; il forme un apport interne, implicite et précoce, qui détermine une première fois le référent thématisé : dans l'exemple le référent de when est le moment d'occurrence de his abstraction had become something more than natural et de nul autre procès; il est inconnu mais ce rhème le rend défini (unique dans sa catégorie). Ce genre d'apport existe dès qu'un référent est thématisé dans une proposition; il fait de la participation au procès une propriété distinctive et contribue à l'identité interne (cf. 3 .3.1.); comme on a vu, c'est un apport intégré nécessaire à la question («le moment d'occurrence de ce procès-là, c'est cela que je veux savoir »). Le second apport est celui externe, ainsi préparé, de la réponse-propos attendue ; il a vocation à remplacer le mot interrogatif dans la fonction qui est la sienne dans la proposition interrogative, c'est pourquoi, si cette fonction est circonstancielle, il a la forme d'un adverbe ou d'un SPrep (cf. after their clash over Anthony Browne). Dans cette analyse le référent thématisé est deux fois support et les apports sont hiérarchisés; il y a question quand le référent a intégré l'apport subordonné mais substrat du rhème et qu'il attend au premier plan énonciatif l'apport supérieur d'une réponse.

3) En grammaire systémique fonctionnelle, le thème est "point de départ d'un message "; implicitement sa première position est iconique de cette fonction. Sans contradiction, on peut dire aussi, dans la perspective de la relation support-apport, qu'un référent posé premier n'est relatif à aucun autre dans l'unité qu'il commence alors que ce qui le suit doit lui être relatif; l'apport doit être second et le support premier. Dans l'exemple du texte but précède when cependant (cf. 1.2.) : le mot en wh et seulement tenu de commencer la partie référentielle de l'énoncé qui réunit procès, participants et circonstants ; cette condition satisfaite, il peut être précédé ou parfois suivi de morphèmes relevant des composantes textuelle ou interpersonnelle du discours. Dans leur analyse des interrogatives, Halliday et Matthiessen citent des continuatifs (well, yes, no, oh, now), des conjonctions (and, but, then), des adjoints continuatifs (in that case, to be precise), des adjoints de commentaire modal (surely, in your opinion...), des vocatifs $(2004,79-81)$; ex. inventé : Well, but in that case, in your opinion Iris, when...?

\subsubsection{Fonctionnement du mouvement de wh interrogatif en lien avec la thématisation}

Dans une phrase complexe la question peut thématiser un participant/circonstant de la principale (4a) ou d'une subordonnée ( $4 \mathrm{~b}$ ) ; $4 \mathrm{c}$ est la version in situ de $4 \mathrm{~b}$ :

(4) a. Who thought that his abstraction had become something more than natural ?

b. And when do you think that his abstraction became something more than natural

-?

c. You think that his abstraction became something more than natural when ? 

tête de la proposition où il est circonstant (cf. $4 \mathrm{~d}$ ) mais en tête de la principale :

(4) d. *You think that when did his abstraction become something more than natural?

Parce qu'une question partielle thématise un référent au-dessus de tous les autres dans un énoncé, si une avancée explicitante et expressive a lieu, elle porte naturellement le wh y renvoyant en tête de phrase. La logique de la thématisation contredit alors celle des relations sémantico-référentielles. Un constituant syntaxique renvoyant à un participant d'un procès est naturellement dans la proposition signifiant ce procès ; or quand il est avancé dans une question, comme en $4 \mathrm{~b}$, il est détaché de la subordonnée à laquelle il appartient, où un constituant canonique n'est plus réalisé, et il est rattaché à la principale, où il n'est ni complément ni circonstant, au risque de l'ambiguïté. La force de la composante pragmatique des questions se mesure là.

L'énoncé 4e montre une avancée du sujet de la subordonnée :

(4) e. What do you think - happened to him ? emploi est marqué: typique des interrogatoires, il est caractérisé par une forte accentuation et par l'insistance. Son expressivité est de saillance. 1) L'interrogative à wh in situ construit la question plus directement que celle à wh avancé puisqu'elle remplace seulement le constituant indéfini préconstruit par wh ; ainsi elle peut traduire une hâte de savoir. 2) Ce court chemin laisse aussi la déclarative sous-jacente reconnaissable dans l'interrogative ; ainsi ce que l'énonciateur veut savoir (l'identité du référent de l'interrogatif) contraste-t-il dans le linéaire avec le contenu acquis de cette déclarative, comme une forme détachée d'un fond, et le constituant interrogatif, qui est généralement en fin d'énoncé de surcroît, est-il d'autant plus focalisé. Par comparaison, l'expressivité du mouvement de wh est de convergence ou d'harmonie : l'avancée joue et montre la thématisation signifiée par wh.

\section{La thématisation dans les subordonnées interrogatives}

\subsection{Impossibilité de wh interrogatif in situ}

Dans une subordonnée interrogative wh doit être le premier constituant référentiel. En $5 \mathrm{a}$ who, attribut du sujet, n'occupe pas la position de complément de be; $5 \mathrm{~b}$, où il est in situ, est agrammatical (cf. aussi 5c-d).

(5) a. Another day he asked her suddenly who Rosemary's best women friends had been -. 
b. *Another day he asked her Rosemary's best women friends had been who.

c. Iris wondered when exactly his abstraction had become something more than natural -.

d. *Iris wondered his abstraction had become something more than natural when exactly.

51 Sans doute les phrases à interrogative subordonnée qui signifient une question indirecte ou rapportée, sont-elles moins propices énonciativement à la saillance des wh in situ que les questions directes et se satisfont-elles en revanche de l'expressivité plus abstraite de la première position. Mais la subordination explique aussi la contrainte.

Un verbe interrogatif (wonder, ask) ou un prédicat à interprétation interrogative (be a mystery) donne à un argument le rôle sémantique "stimulus » ou "contenu de la question » (v. infra) ; une phrase complexe comme 5 a ou c résulte de l'instanciation de cette position d'argument par une proposition en wh. Dans une telle proposition le référent de wh a la fonction pragmatique "thème interrogatif ", qui lui vaut d'être support et de recevoir l'apport rhématique de la proposition, ainsi attachée à lui (cf. 3.5.1). De façon naturelle c'est ce référent qui instancie la position de l'argument évoqué dans la proposition supérieure; il devient complément nominal du prédicat interrogatif, entraînant dans cette fonction la proposition qui est son apport. Dans cette opération, la proposition interrogative est subordonnée au verbe principal indirectement, en étant subordonnée directement à wh, son support intrapropositionnel; de son côté, wh est capable de subordonner sa proposition parce qu'il y est thème et qu'il est pronom (au moins en partie), c'est-à-dire qu'il est susceptible d'avoir un apport et une fonction nominale dans une unité supérieure. La hiérarchie support-apport, interne à la proposition interrogative, a un rôle décisif dans la subordination externe de la proposition au prédicat supérieur et dans la construction de la phrase complexe; c'est pourquoi elle est explicitée syntagmatiquement par l'avancée et par la position première de wh. Premier, wh est démarcatif. Étant thème, il indique dès qu'il apparaît qu'il est le support d'une unité nouvelle ; étant pronominal, il indique que cette unité n'est pas une proposition principale mais qu'elle a un rôle nominal, subordonné, dans une unité plus grande.

\subsection{Subordonnée objet d'un verbe et subordonnée complément d'un nom}

53 Verbe de parole, ask a dans sa structure argumentale un argument «message ». Quand ce qui est demandé n'est pas une action (ask to do something) mais une information, cet argument est subdivisé en "message label" (nom de type illocutoire ; ex : question) et “ message content" selon Dixon (2005 : 146-156). Dans une phrase, cet argument peut être instancié par le type illocutoire (she asked him a question), le contenu (she asked him what was the matter), ou les deux réunis en un SN complexe:

(5) e. She asked him a question as to what was the matter.

f. She asked him the question what was the matter.

Dans ce dernier cas, type illocutoire et contenu du message sont hiérarchisés : le nom abstrait de type illocutoire est tête du SN et le contenu particulier du message est son complément. Avec un argument "message" simplifié, question a la même structure argumentale que ask (cf. a question from $x$ to $y$ about $z$ ). À l'instar de ce verbe, dont il est un double nominal, il peut aussi renvoyer à la question globalement, appréhendée comme un événement borné, situé, mettant en jeu des relations interpersonnelles et 
pouvant s'inscrire dans une trame événementielle; après ask il n'est cependant pas redondant : il signifie que l'objet de la demande est une information.

En (5e) la question est une référence nouvelle focalisée; le nom de type illocutoire question est indéfini et il est mis en relation explicitement avec son argument « contenu de la question " par la préposition complexe as to. Cette préposition, qui gouverne wh + $p$ signifie une visée : la sélection par la question d'un contenu particulier; présentative, elle annonce et focalise ce contenu dans le linéaire. La préposition est obligatoire en (5e), où l'indéfini signifie une première présentation du référent; elle est interdite en (5f), où le référent est défini, même s'il est mentionné pour la première fois. Le défini signifie que son contenu particulier rend la question unique dans sa catégorie et qu'il est intégré à son identité. Il implique que la relation question-contenu n'est plus en construction mais qu'elle est acquise; la préposition as to, qui lie des entités qu'elle présente comme distinctes, n'est plus de saison et l'interrogative instancie l'argument « contenu » de question directement.

Dans l'exemple 1.13-14, le SN figure her question as to what was the matter:

(5) g. to her question as to what was the matter, he replied briefly, 'Nothing'.

57 Le possessif porte le trait « défini »; cependant la question est unique pour être celle posée, comme montre l'équivalence her question = the question that she asked, non à cause de son contenu. Le rapport question-contenu est construit dans l'énoncé et la préposition est naturelle. Le SN équivaut à la proposition her question was "what was the matter" ; la préposition as to explicite la relation question-contenu, comme be le fait, et elle focalise le contenu comme il peut l'être dans la proposition; tout en faisant cela, elle permet une expression non propositionnelle de la relation.

Le verbe reply a deux arguments "messages": un argument "réponse ", limité à un contenu, réalisé canoniquement par un complément central proche du verbe, et un argument "question », qui compte nom de type illocutoire et contenu, réalisé par un complément non central, introduit par to et plus éloigné du verbe. Les propriétés grammaticales citées reflètent le fait que l'acte de répondre apparait étroitement lié au contenu qu'il produit, mais distinct de l'acte interrogatif qui le suscite. Dans le second complément, la préposition to signifie que dans la paire adjacente question/réponse, la question, première établie, est repère (elle porte le thème interrogatif) et que la réponse, seconde et repérée, lui est relative (constituant un propos/apport); métaphoriquement elle indique leur écart et l'application de la seconde à la première.

En (5g), le complément non central est antéposé. L'avancée de la référence à la question superpose l'ordre du texte à celui des événements et rend la séquence iconique ; elle souligne aussi que la question est le cadre énonciatif de la réponse et elle rend la séquence prospective: l'avancée d'une dépendance, marquée telle d'entrée par la préposition to, crée l'attente du sujet et du prédicat auquel cette dépendance se rattache et elle prépare la focalisation du second. Dans le constituant référant à la question, la partie "contenu» est focalisée par as to (v. supra), mais l'orientation prospective générale de la séquence se traduit par une focalisation plus forte de la réponse. La réponse est citée au style direct ('Nothing') alors que la question est rapportée au style indirect; la fin de la séquence nous met sur la scène énonciative et dans le présent de la réponse.

60 En $(5 \mathrm{~g})$ le pronom indéfini nothing instancie la position « réponse » de reply. Séparé par une virgule et entre guillemets, il cite le contenu d'une réponse dont l'existence est 
affirmée dans la proposition et supposée par l'adverbe briefly, qui la décrit. Contenu d'une réponse, nothing s'applique sémantiquement à la question référée en $(5 \mathrm{~g})$; il " remplace » le what interrogatif thématisé de what was the matter ainsi que le something sous-jacent, pour construire un énoncé déclaratif négatif qui réfute le présupposé de la question. Le verbe answer permet le contraste answer nothing vs answer, 'Nothing'; la première occurrence du pronom détermine le procès "answer» et niant l'acte locutoire, nie l'acte de langage; la seconde occurrence, citant une réponse, suppose l'existence de l'acte « répondre » et détermine la proposition interrogative.

\subsection{Les cas de double niveau de subordination}

61 La phrase complexe (5h) montre deux niveaux de subordination propositionnelle : dans la principale, think a pour complément interne la proposition complexe she saw what was in his mind, qui lui est subordonnée ; dans celle-ci, see a pour complément interne l'interrogative what was in his mind, également subordonnée :

(5) h. She thought she saw what was in his mind.

62 La subordination inclut un constituant dans un autre, qui le régit. En (5h), la subordination des compléments propositionnels se trouve exprimer cette «inclusion intentionnelle (au sens technique du mot) de la chose pensée dans la pensée » qui est la marque du mental pour la phénoménologie. Dans see what was in his mind le verbe see implique que what was in his mind tombe sous les opérations d'une pensée; de même dans she thought she saw what was in his mind, think implique que she saw $+p$ est objet de pensée.

63 Les procès signifiés forment une chronologie, qui permet une analyse génétique, où la hiérarchie syntaxique est construite du bas vers le haut, la proposition la plus subordonnée étant première en genèse et la principale, dernière. Dans cette construction, l'interrogation sur l'identité de wh, what was in his mind, est première ; c'est le préconstruit de she saw what was in his mind, la compréhension de ce qu'est wh, qui est le préconstruit de she thought she saw what was in his mind, le jugement de la compréhension. Il y a enveloppement de what was in his mind par la proposition de see, puis de she saw what was in his mind par celle de think; chaque enveloppement produisant une unité syntaxique supérieure.

Dans l'opération d'inclusion, le préconstruit donne l'impulsion: l'interrogative what was in his mind suscite une recherche sur le thème interrogatif, que see implique; la perception intellectuelle she saw $+p$ appelle le jugement, d'Iris ou du narrateur, qui la nuance (think). En convoquant un procès cognitif où il a vocation à être "stimulus ", le préconstruit entre en relation avec un "expérient» dans le champ de ce procès; de cette inclusion la traduction syntaxique est la subordination.

See signifie le succès de la recherche suscitée par l'interrogative; la réponse n'étant pas dévoilée, le procès cognitif n'est pas le propos appelé par la thématisation de wh, mais le procès est bien repéré en fonction du préconstruit, son repère, et il est un apport pour lui. De même think signifie une métacompréhension qui poursuit et achève la compréhension de see; la perception intellectuelle est le repère du jugement, qui lui apporte une détermination modale.

66 Dans la genèse, la proposition signifiant la dernière unité créée est la "principale ", qui est coextensive à la phrase. C'est elle qui reçoit le mode. Le mode concerne tout 
l'énoncé et il est porté naturellement par cette proposition; la position finale que lui reconnaît l'analyse génétique apparaît également naturelle.

La genèse proposée illustre la «successivité verticale» et l'inversion de l'ordre génétique de production par l'ordre linéaire de rangement dont parle Guillaume (cf. 2). L'inversion dépend du verbe supérieur. Si au préconstruit what was in his mind est appliqué be a mystery, dans la phrase résultant, l'ordre du linéaire reflète celui de la genèse (what was in his mind is/was a mystery). Les verbes see et think, en revanche, mettent en relation un expérient, siège de pensée, et un stimulus, chose pensée, qui doit être leur complément interne à l'actif; comme what was in his mind instancie l'argument stimulus de see, la phrase se développe à gauche de l'interrogative, et ce qui est premier dans le linéaire est dernier en genèse.

Presque tous les verbes de perception et la plupart des verbes de jugement ont à l'actif l'expérient pour sujet et le stimulus pour objet. Cette distribution, qui est à l'origine de l'inversion décrite, est peut-être motivée. Dans la perception ou le jugement la chose perçue ou jugée est un "objet de pensée ", inclus dans l'esprit qui pense : 1.26 Iris accède à une représentation, dans son propre esprit, de ce qui est dans l'esprit de George ; au contraire, celui qui perçoit ou qui juge inclut l'objet pensé dans son esprit et il a la conscience de cette inclusion : il inclut sans être inclus.

Syntaxiquement, l'objet et le sujet sont des compléments du verbe ; l'objet, complément interne, peut porter des marques de subordination au verbe, alors que le sujet, complément externe, n'en montre pas. La subordination est une inclusion: l'alignement du stimulus avec le complément interne peut s'expliquer par le partage de ce trait.

Dans un autre contexte what was in his mind pourrait être un SN à proposition relative nominale. L'énoncé she hated what was in his mind signifie une appréciation d'un objet identifié. Les différences syntaxiques entre interrogatives et relatives nominales sont connues; on peut penser que dans les relatives, nominales ou adjectivales, la position de tête du wh exprime aussi une thématisation.

Dans une proposition indépendante signifiant un procès, une entité nominale est conçue principalement comme un participant de ce procès. Cette participation, acquise, peut être inscrite en elle comme propriété distinctive. Ce qui réfère à l'entité ainsi enrichie est un $\mathrm{SN}$ à relative où la proposition signifiant le procès est une dépendance/ un apport (what was in his mind). Le SN résulte d'une nominalisation interne qui thématise l'entité en cause afin qu'elle soit le support de la proposition, et l'avancée du relatif en tête de proposition traduit cette thématisation (Cotte, 1997 : 271-302). Cette analyse permet d'expliquer pourquoi, dans certaines langues, les pronoms interrogatifs ont acquis un emploi de pronom relatif.

\section{Incidence de la thématisation sur Aux (temps)}

Dans une principale ou une indépendante, où la question «directe " est au premier plan énonciatif, si wh a été avancé en tête, le temps doit être avant le sujet, quitte à ce qu'un auxiliaire soit introduit pour le porter et au prix d'un second mouvement syntaxique qui produit la structure WH Aux (temps) Sujet Prédicat.

(6) a. But when exactly had his abstraction become something more than natural ? 

sujet :

(6) b. What do you think (that) he wanted _ ?

c. Who do you think _ told her?

Ainsi dans Who told her? l'interrogatif sujet who n'est pas nécessairement suivi de Aux (temps) pour l'unique raison que ce constituant n'a pas été avancé en tête de l'interrogative comme il l'est en (6c). Les exemples (6b) et (6c) montrent que l'auxiliaire après wh porte le temps de la principale (le présent) et non celui de la subordonnée dont wh est extrait; les séquences (6d) et (6e), où Aux porte le prétérit de la subordonnée, le verbe principal restant au présent, sont agrammaticales :

(6) d. *What did you think (présent) that he want (inf) _ ?

e. *Who did you think (présent) _ tell (inf) her ?

On fait l'hypothèse que l'« inversion du sujet et de l'auxiliaire » est expressive, comme le mouvement de wh qui en est l'occasion : elle exprime une thématisation secondaire préparatoire.

Un verbe auxiliaire a un verbe lexical ou auxiliaire pour complément. Régissant un autre verbe, il est tête, et s'il n'est pas régi par un autre auxiliaire, il est tête du Syntagme Verbal (prédicat). À ce titre, il peut prendre la détermination du temps, qui vaut pour la proposition; il peut mettre le prédicat en relation avec le sujet (accord) et il peut le représenter dans certaines opérations. On admet que l'Aux qui s'inverse avec le sujet représente le prédicat de la proposition et le procès qui y est signifié par le verbe lexical.

77 Dans une proposition dont le procès est présupposé, une question en wh thématise le référent du mot interrogatif; elle ne le fait pas sans thématiser aussi le procès, qui est nécessaire à la compréhension de la question et que la réponse est destinée à enrichir.

Dans la construction de sa question l'énonciateur prend donc pour thème large (cadre) his abstraction had become something more than natural et dans ce cadre il prend pour thème étroit (support) le moment du procès. Implicitement il dit: «Je parle de ce procès; dans ce procès je parle du moment; je demande ce qu'il est ». L'analyse du contexte de (6a) en 1. a montré cette séquence thème large (cadre) --- thème étroit (support); les mêmes opérations sont à l'échelle du texte et dans la genèse de la question. Parce qu'elle est impliquée par la thématisation en wh, la thématisation du procès reste implicite dans nombre d'interrogatives, mais elle est signifiée dans les questions « directes ", qui sont plus expressives.

Comme il est suggéré, la thématisation du procès est première et celle en wh est seconde dans la genèse de la question. Chaque thématisation porte un nouveau thème en tête et crée une nouvelle unité. L'unité précédente restant dans le préconstruit, il en résulte une structure hiérarchisée, dans laquelle le sujet de la proposition est thème initial, Aux est thème intermédiaire préparatoire, et wh est thème final, et principal, de la question.

80 Remarques : 1) Sans la position fixe du sujet dans la proposition, les thématisations d'Aux et de wh ne seraient pas perceptibles. 2) L'« inversion du sujet et de l'auxiliaire " est en réalité l'antéposition d'Aux, représentant du procès, au sujet. 3) La thématisation de wh à partir de l'unité où Aux est thème correspond à l'«extraction" de ce constituant (en $6 \mathrm{~b}-\mathrm{c}$ wh est extrait du prédicat où se trouve sa proposition d'origine). 4) La genèse montre une successivité verticale et une inversion de l'ordre génétique par

Corela, HS-29 | 2019 
l'ordre linéaire à nouveau; cette inversion est motivée différemment de la première étudiée.

\section{Les interrogatives clivées : l'interprétant}

81

(the moment) when his abstraction had become something more than natural. Dans cette transformation, l'adverbe relatif when, comme l'adverbe interrogatif de même forme, reprend le SPrep et thématise le moment du procès dans la proposition. Cette thématisation, qui exige l'avancée en première position du wh, n'institue pas le moment thème pour un propos extérieur à la proposition; elle l'institue tête, et support, d'une structure nominale nouvelle, où la proposition initiale, transformée en relative, est son apport. L'apport est la participation du moment au procès; cette participation est maintenant une propriété distinctive intégrée. Enrichi par l'apport, le moment, qui est indéfini et quelconque dans la proposition initiale, est rendu défini (the moment when $p$ ) ; il est unique pour être « le moment de ce procès-là »-formule où l'on reconnaît l'« identité interne » de la précédente analyse.

3) À ce stade l'identité externe est absente : le SN entre comme sujet (thème) dans une proposition dont le prédicat (propos) est susceptible de dire cette identité. Dans cette proposition be pose une correspondance entre le référent du sujet «identifié » et celui du complément du sujet «identifiant »; le premier, donné, est thématisé ; le second, nouveau, est focalisé : (the moment) when his abstraction had become something more than natural was $X$; quand le sujet a wh pour tête, la syntaxe générative parle de "pseudoclivée ».

4) La phrase clivée est dérivée de cette phrase en be. Elle substitue it au sujet complexe (the moment when $p$ ) afin d'introduire l'identifiant plus directement et d'augmenter sa focalisation. Dans la dérivation, le pronom it reprend et remplace le SN sujet de la pseudo-clivée. Courante dans les textes, cette anaphore a lieu ici dans la genèse d'un énoncé. It reprend seulement le trait "défini»: il signifie que le référent est présupposé et unique, mais il occulte sa catégorie nominale et la propriété distinctive qui le rend unique. Sans place dans le sujet, dès lors que it est tête, et sans rattachement logique au prédicat, la relative est extraposée : elle prend place hors de la proposition que le prédicat clôt; toutefois sa position étant fonction de celle des autres constituants, elle reste dans la phrase. 
(7) c. It was X that his abstraction had become something more than natural _. un contraste entre un arrière-plan occupé par la relative (généralement en that) et la proposition initiale sous-jacente, typiquement présupposée ${ }^{9}$, et un premier plan, porteur du mode, dédié à l'identification du sujet par le complément interne de be. Ainsi, il isole et met en relief (focalise) cette identification, pour signifier que l'identité externe du référent du sujet, qui est nouvelle (ou ignorée), compte plus que l'identité interne, qui est donnée. À l'intérieur de la proposition le choix de it est focalisant aussi. La réduction sémantique du sujet crée un contraste syntagmatique "peu informatif/ très informatif » où le second terme, saillant, concentre l'attention, tandis que le premier prend un rôle présentatif ; c'est ainsi que (7a) introduit dynamiquement, en la focalisant, la réponse à la question en when. Dans la pseudo-clivée au contraire, le sujet, informatif et long, retient plus l'attention et il retarde l'introduction de l'identifiant. Dans les pseudo-clivées et dans les clivées, le complément interne de be est interprété comme unique identifiant possible du sujet; la stratégie focalisante de la clivée le souligne.

5) Dans la clivée interrogative (7b), aucune identité externe possible n'étant sélectionnée, le complément interne de be est remplacé par un interrogatif en wh (It was when that $p$ ?). Wh appréhende l'identité externe abstraitement, comme une variable, il la thématise et il donne l'instruction à l'allocutaire de sélectionner la bonne valeur lors d'un parcours des valeurs possibles. Au lieu de poser et de focaliser une identité particulière, comme fait (7a), la clivée-interrogative focalise donc la thématisation de la variable, c'est-à-dire l'opération interrogative, qui continue la construction du sens audessus de la proposition en be. When passe en tête d'énoncé pour exprimer la thématisation interrogative; tête du prédicat dont wh est extrait et porteur du temps grammatical, be et avancé aussi (cf. 5.).

89 Les interrogatives clivées sont équivalentes sémantiquement aux non clivées. Rares à l'écrit, elles sont familières à l'oral (What is it you teach ?) ou elles sont marquées. Elles sont fréquentes dans les devinettes parce qu'elles séparent de la question proprement dite la partie qui dit, outre l'identité interne du sujet, les propriétés qui sont censées faire deviner. Cette partie, qui correspond à la relative, est nouvelle pour l'allocutaire et elle est focalisée en fin d'énoncé (What is it that no one wants but no one wants to lose ?).

Dans les questions portant sur une variable, la construction du sens compte plus d'opérations que ne donnent à penser les marques grammaticales, finalement peu nombreuses, des interrogatives non clivées. L'écart entre les marqueurs et les opérations s'explique par le caractère logique de certaines opérations. Dans la construction du sens, si une opération est la condition nécessaire d'une autre, signifier la seconde dispense de signifier la première : s'il est nécessaire qu'un référent ait une première identité connue des co-énonciateurs pour que son identité externe soit l'objet d'un échange entre eux, une question en wh à propos de cette dernière, supposant acquise la première identité, peut ne coder que l'opération seconde signifiée par wh. C'est ainsi que la question en wh non clivée a une genèse courte, de la proposition initiale his abstraction had become something more than natural jusqu'au mouvement de wh, alors que la clivée correspondante a une genèse longue entre les deux mêmes termes.

91 Chaque genèse est cependant indépendante de l'autre : la question non clivée ne résulte pas de l'ellipse de parties de la clivée, ni celle-ci d'un étoffement interne de celle-là. En 
revanche, l'interrogative clivée, explicitant des étapes de toute question à propos d'une variable, est interprétable comme une réélaboration didactique et expressive de l'interrogative simple, comme une structure à propos d'une structure.

Les principaux faits qu'elle souligne sont: 1) Une question en wh demande qu'un référent soit identifié ; la proposition en it le dit explicitement (it/be). 2) L'entité sur laquelle porte l'interrogation est complexe et construite dans l'interrogation. Cette entité est membre d'une catégorie; participant d'un procès, elle reçoit de sa participation une identité interne qui la stabilise (toute question en wh contient ainsi une relative, qui est secrète dans les non-clivées) et elle a nécessairement une identité externe, qui est cherchée. La clivée montre cette complexité grâce à la relative et à des saisies distinctes, par it et par wh, du référent. 3) La question est le produit de thématisations successives. 4) La thématisation interrogative, principale, est focalisée (it).

Il est possible de considérer que la question clivée, qui dit comment l'interrogative simple signifie la question tout en signifiant la même question qu'elle, est, en empruntant à la sémiotique de Peirce (1931-1935, vol. $4: 132)$, un « interprétant ».

\section{Les questions polaires}

\subsection{Identification de l'opération de thématisation}

L'énoncé 8a est une question polaire, qui présuppose la vérité de la disjonction Rosemary talked to you much or she didn't talk to you much, et dont la réponse est censée être yes/no.

\section{(8) a. Did Rosemary ever talk to you much ?}

Le préconstruit est Rosemary talked to you [much] sometimes. Dans cette proposition, le procès Rosemary talked to you est présupposé, mais l'énonciateur ne sait pas si talk much, avec ses connotations qualitatives, est vrai. La proposition préconstruite n'est donc pas présupposée ; c'est une proposition logique, une "entité de $3^{\mathrm{e}}$ ordre " pour Lyons (1977 : 445) dont la valeur de vérité, inconnue, est en question.

L'énonciateur espère que la proposition est vraie, mais l'interrogative aurait la même grammaire si sa valuation du procès était défavorable. Sa question est «neutre »: il n'indique pas quelle réponse il juge probable, ni ne donne à penser que quelque élément de la situation contredit un premier jugement.

Dans la genèse de (8a), l'énonciateur thématise la proposition et la polarité positive. Il suspend celle-ci provisoirement, faisant de la catégorie de la polarité une variable dont il parcourt les deux valeurs sans succès. Il demande à l'allocutaire qu'il sélectionne l'une d'elles, après un parcours des moments d'occurrence possibles du procès (ever), et qu'il confirme ou infirme la polarité thématisée. Comme dans les questions en wh, la thématisation est double; la glose est: "Dans le cadre de cette proposition (thème large) je demande (propos) que la polarité positive (thème étroit) soit confirmée ou infirmée (réponse) ». 


\subsection{Polarité neutre et polarité négative} polarité :
Les questions polaires neutres thématisent généralement le positif; (8b) est une question neutre douteuse :

(8) b. ${ }^{(*)}$ Didn't Rosemary (ever) talk to you much ?

La logique n'interdit pas la polarité négative, car il revient au même que la réponse affirme ou nie le positif, ou qu'elle nie ou affirme le négatif. La polarité positive représente l'application de la proposition au monde, qui est l'enjeu de la question polaire neutre ; il est naturel, et suffisant, de soumettre cette application au jugement du co-énonciateur, alors que la réponse doit l'affirmer ou la nier explicitement.

Les unités linguistiques, petites comme le lexème talk, ou grandes comme la proposition dont ce verbe est la tête, ont pour propriété l'« applicabilité » (Lyons, 1977 : 213-4), la capacité, et la vocation, à s'appliquer au monde. Cette propriété explique que la polarité positive, à la différence de la négative, n'ait pas besoin de marque ; elle explique aussi que les déclaratives positives, qui affirment que leur proposition s'applique au monde, aient la grammaire la plus simple ; ces propositions réalisent le programme de leurs signes.

Un emploi neutre de la polarité négative s'observe cependant dans certaines questions alternatives, où l'énonciateur, typiquement, presse l'allocutaire d'indiquer la bonne

(8) c. Did she or didn't she/did she not talk to you?

Dans une telle question, l'énonciateur parcourt explicitement les deux polarités de la proposition ; il thématise chacune et il demande à l'allocutaire non de confirmer ou d'infirmer (yes/no n'apparaît pas normalement dans la réponse) mais de choisir et d'asserter la bonne polarité. Le parcours confirme la priorité de la polarité positive, qui est thématisée avant la négative.

\subsection{L'inversion auxiliaire-sujet}

Dans les questions polaires neutres, principales ou indépendantes, la thématisation de la proposition et de la polarité positive est signifiée par l'antéposition au sujet de l'auxiliaire tête conjugué, dont le reste du prédicat est le complément interne. Cette opération, au premier plan énonciatif, laisse à l'arrière-plan la proposition logique, qui est préconstruite.

4 En l'absence d'auxiliaire indépendamment motivé, l'auxiliaire est do (cf. 8a). Auxiliaire du mode, celui-ci exploite une propriété du do lexical dont il est issu. Un procès de type état est sans bornes notionnelles; un procès dynamique a des bornes notionnelles et il implique une opposition entre un intérieur positif et un extérieur négatif ; enfin une occurrence de procès, statique ou dynamique, est bornée aussi et implique également une opposition positif/négatif. Le lexème verbal do signifie un procès dynamique. L'auxiliaire issu de lui retient l'opposition du positif et du négatif et il représente abstraitement l'occurrence positive du procès, dynamique ou statique, qui est signifié par son complément interne. Cette occurrence positive, opposée à son contraire négatif, est l'objet des opérations du mode.

7.4. Le cas des question tags 


\section{L'énoncé (8d) (I. 94-5) comporte une proposition déclarative positive et un tag interrogatif « de confirmation » à polarité inverse :}

(8) d. Rosemary saw rather a lot of Anthony Browne, didn't she?

Le préconstruit est la proposition positive prédiquée Rosemary saw (rather) a lot of Anthony Browne; elle comporte une partie connue présupposée, R. saw A.B., et une quantification subjective nouvelle, rather a lot. L'énonciateur affirme cette proposition, puis il pose une question.

Le tag montre seulement les constituants et la syntaxe nécessaires à une question polaire. Il ne contient pas de proposition à laquelle sa question s'appliquerait ; son sujet est anaphorique de celui de la proposition affirmée ; son auxiliaire, s'il ne répète pas celui de la proposition, n'ajoute pas au sens. Le tag est donc joint à la proposition qui précède, dont il est un « supplément » et sa question, comme l'assertion, s'applique à la proposition préconstruite de l'énoncé.

108 Énonciativement, il ne sert pas principalement à atténuer ou à renforcer l'assertion, quoique ces effets existent parfois, mais il invite l'allocutaire à partager (co-valider) cette assertion. Il contribue à l'ajustement des co-énonciateurs; dans le texte, il prélude à d'autres questions sur les relations entre Rosemary et Anthony Browne.

109 Après avoir asserté la proposition positive préconstruite, l'énonciateur considère la proposition négative contraire. Il la met en question; son énoncé montre alors sa préférence en contrastant la polarité assertée avec celle discutée secondairement.

110 Le tag construit aussi cette préférence. Asserter une polarité exclut la polarité inverse. Thématiser une polarité et demander à l'allocutaire s'il l'asserte ou non, c'est ne pas l'asserter soi-même, et c'est permettre qu'elle soit concurrencée par la polarité opposée, non écartée. La thématisation interrogative suscite un parcours où les polarités sont égales logiquement: dans le tag, aucune n'est assertée et chacune est concurrencée par l'autre. Cependant, elle fixe au premier plan énonciatif, en tant que cible de la question, une seule des polarités non assertées et concurrencées (la négative en $8 \mathrm{~d}$ ) et elle la fait apparaître comme l'objet d'un doute de l'énonciateur, tandis que celle non mentionnée est, par contraste et implicitement, préférée.

111 C'est ainsi que le question tag de (8d) est orienté vers une réponse positive. L'énonciateur demande à l'allocutaire s'il asserte ou non le négatif thématisé, mais il se montre disposé à une référence positive qui confirmerait l'assertion par laquelle son énoncé commence. L'allocutaire répond ce qu'il veut, mais un enjeu de sa réponse est de conforter ou de contredire le choix implicite de l'énonciateur. L'intonation du tag indique si celui-ci préjuge de son accord.

\subsection{Le cas des énoncés interro-négatifs}

112 L'énoncé interro-négatif (8e) demande l'avis de l'allocutaire plus nettement que le tag de (8d), mais la logique de la question et son orientation sont les mêmes :

(8) e. Didn't Rosemary see rather a lot of Anthony Browne?

113 Dans cet énoncé, l'énonciateur pense que Rosemary saw rather a lot of Anthony Browne est vrai. Sans affirmer cette proposition lui-même, il crée les conditions pour que l'allocutaire le fasse. Comme dans le tag de $(8 \mathrm{~d})$, il thématise la polarité négative de Rosemary did not see a lot of Anthony Browne et il interroge l'allocutaire à son sujet ; il ne 
tranche pas entre les polarités mises en concurrence, mais comme précédemment, en thématisant le seul négatif, il montre qu'il doute de cette polarité et qu'il pense que le positif est vrai.

Dans d'autres contextes, une interro-négative peut exprimer un conflit. L'énoncé (8e) peut alors se gloser : 1) Je pensais que Rosemary saw a lot of Anthony Browne était vrai ; 2) selon ce que vous dites ou ce que je découvre, la proposition négative Rosemary did not see a lot of Anthony Browne est vraie ; 3) je ne sais que penser et je vous demande, en thématisant dans ma question la polarité négative inattendue, si vous la confirmez. Une autre situation est : 1) J'avais dit que la proposition positive était vraie ; 2) vous aviez répondu que la proposition négative était vraie ; 3) il apparaît que j'avais raison ; 4) je vous demande, en thématisant dans ma question la polarité négative que vous avez assertée, si vous maintenez encore celle-ci. Avec une prosodie chaque fois adaptée, d'autres possibilités existent ; la constante des questions négatives est de thématiser la polarité négative. On doit noter que les interrogatives positives, outre qu'elles forment les questions neutres, ont des emplois symétriques en tout point de ceux étudiés en 7.4. dans les tags et ailleurs, même si ce fait n'est guère reconnu.

\subsection{Le cas des « questions déclaratives »}

Une autre demande de confirmation est illustrée par la séquence 8f, qui comporte deux « questions déclaratives » (Huddleston \& Pullum 2002 : 881-2) :

(8) f. But she hadn't known him very long - he was more or less of a casual acquaintance?

Chaque proposition est déclarative et interrogative: sa syntaxe est celle du mode déclaratif; son intonation, ascendante à la fin, est celle d'une question polaire. Comme dans une proposition à laquelle est joint un tag à polarité inverse, le mode déclaratif asserte la proposition préconstruite, en excluant une des polarités, tandis que la question demande à l'allocutaire s'il partage / de partager l'assertion en rétablissant la polarité exclue comme option logique. En première analyse (8f) équivaut donc à $(8 \mathrm{~g})$ :

(8) g. But she hadn't known him very long, had she?; he was more or less of a

casual acquaintance, wasn't he?

117 Mais une différence existe. Un question tag à polarité inverse invite à confirmer la polarité d'une proposition assertée de manière indirecte, en thématisant la polarité contraire (v. supra). Son mouvement dialectique favorise une réponse explicite, qui rétablit la bonne polarité contre la mauvaise thématisée, comme le texte le montre aux lignes 96 et 99 . De son côté, une question déclarative invite à confirmer la polarité assertée directement. Signifiée par l'intonation, la question est réalisée pendant l'assertion, sur le seul matériau lexical de la proposition; elle thématise et soumet à l'avis de l'allocutaire la polarité qui y est assertée, non son opposée, qui n'est qu'implicite. L'énonciateur la préfère au question tag quand il ne donne pas un tour argumentatif à sa question et qu'il est prêt à se satisfaire, le cas échéant, d'un accord tacite, ce que le texte montre également. Ainsi les deux questions déclaratives de (8f) forment-elles une séquence plus fluide que les deux énoncés avec tag de (8g) : en (8f), mais pas en $(8 \mathrm{~g})$, la seconde question peut être interprétée comme un supplément de la première et la séquence comme un seul énoncé exigeant un seul point d'interrogation ; enfin, en (8f), l'énoncé qui suit la seconde question déclarative s'enchaîne 
immédiatement, comme montre l'ellipse du sujet de Used to take her dancing, qui serait difficile après $(8 \mathrm{~g})$.

Après didn't he ? 1.98, 'Yes' peut répondre à ce seul question tag; cependant, les trois assertions précédentes formant une seule séquence, ce morphème, que ne suit aucun tag déclaratif, peut signifier aussi une approbation globale.

L'énoncé $(8 \mathrm{~h})$ est une autre question déclarative orientée, une assertive négative à laquelle la prosodie ajoute une demande de confirmation :

(8) h. There wasn't anyone - that you knew of - who - who might have had it in for her?

À propos de la mort de Rosemary, l'énonciateur fait l'hypothèse d'un ennemi dont Iris aurait eu connaissance. Il ignore ce qu'il en est et il pourrait poser une question neutre :

(8) i. Was there anyone that you knew of who might have had it in for her?

121 À la place, il choisit une question déclarative. Il présente son hypothèse à l'allocutaire dans son énoncé ; simultanément, il la nie, parce qu'elle lui paraît osée, effrayante, finalement peu probable, ou qu'il souhaite l'adoucir. Tout en faisant cela, il demande à l'allocutaire de confirmer, ou non, cette assertion négative. Comme précédemment, la polarité assertée est celle thématisée implicitement par la question, la polarité écartée par l'assertion est réintroduite logiquement par la thématisation interrogative ; enfin l'allocutaire signifie son accord tacitement.

122 L'ordre linéaire de l'énoncé ( $8 \mathrm{~h})$ est l'inverse de l'ordre de la genèse. La genèse est : 1) someone might have had it in for her (proposition déclarative signifiant une hypothèse) ; 2) someone who might have had it in for her (nominalisation relative: thématisation de someone, promu tête de $\mathrm{SN}$, transformation de la proposition en relative signifiant une propriété distinctive intégrée à someone) ; 3) there was someone who (...) ( le SN est complément de be dans une phrase signifiant son existence; cette phrase est présentative : elle introduit et focalise le SN en fin d'énoncé) ; 4) assertion négative et question.

À la fin d'un paragraphe relatant la remémoration d'une soirée, l'énoncé (9a) rapporte au discours indirect libre une réflexion d'Iris :

(9) a. Such a gay party it had been, or hadn't it ?

124 L'énoncé a deux parties : une proposition déclarative et un supplément interrogatif s'y rapportant. Elles ont le même sujet, mais leur prédicat est différent. Continuant la description qui précède, la proposition positive affirme la qualité gay; elle s'exclame même sur son haut degré (be such a gay party). En rupture avec elle, le supplément interrogatif introduit la possibilité du contraire (not be such a gay party). Dans le linéaire, l'antéposition de l'attribut de la proposition accentue le contraste avec la polarité du supplément focalisée à la fin de l'énoncé.

Le supplément ne fonctionne pas comme le question tag à polarité inverse de (9b) :

(9) b. Such a gay party it had been, hadn't it ?

L'énoncé (9b) est caractéristique du dialogue. Participant de la même visée énonciative que la proposition assertée, la question, orientée, invite l'allocutaire indirectement à valider la proposition assertée et à confirmer sa polarité positive. Pour sa part, l'énoncé (9a) est caractéristique du monologue intérieur. Le supplément est généralement précédé d'une longue pause. L'énonciateur y exprime une pensée d'après-coup. Après 
réflexion, il se ravise : il commence à s'interroger sur l'opinion qu'il vient d'asserter et à la corriger, pour l'infirmer peut-être.

En (9a), or hadn't it équivaut à or perhaps not. La coordination disjonctive introduit une alternative: la polarité négative, qui est susceptible de se substituer, comme seule vraie, à celle de la proposition. Cette polarité nouvelle est focalisée dans l'énoncé; cependant, à ce stade, elle est seulement possible. La question la thématise sans éliminer l'autre polarité, et elle appelle/annonce le propos qui la justifiera et permettra de l'asserter ; elle est cataphorique, tournée vers la suite du texte.

Dans le tag interrogatif de confirmation (9b), la polarité négative a vocation à être infirmée ; dans le supplément en or, elle est destinée à être confirmée ; mais chaque fois elle est thématisée.

\section{Les questions échos}

Dans la question neutre de 1.48 , George demande à Iris si la proposition thématisée Rosemary said she was afraid of somebody est vraie. Iris répond, mais son propos, qui n'est pas "answer" mais "response", exprime une incompréhension. C'est une question écho, qui reprend la question de George et demande d'expliquer afraid (valeur : « what do you mean?»).

(9) c. 'Did Rosemary ever say she was afraid of anybody?'

'Afraid ?' Iris stared.

'What I'm trying to get at is, did Rosemary have any enemies?'

Pour complexe qu'elle est, la question sur la question est comme les autres. Elle thématise afraid, faisant une variable de cette idée, elle demande à l'allocutaire de lui substituer une valeur particulière et elle attend la réponse qui apportera l'élucidation. Celle-ci, immédiate, a la forme d'une nouvelle question, qui reformule la première et thématise une proposition un peu différente à laquelle Iris puisse répondre.

\section{Conclusion}

131 Cet article a fait l'hypothèse que la signification des énoncés est construite et que cette construction motive leur grammaire; il a présenté une analyse génétique des propositions interrogatives. En syntaxe génétique, une proposition interrogative est une structure stratifiée, dérivée par transformation d'une proposition non interrogative. Chaque étape de la genèse préserve le sens déjà construit et ajoute son opération; dans une telle perspective, le sens interrogatif, au lieu de se résumer à un trait invariant unique, comme le parcours des valeurs d'un variable, est conçu comme un complexe invariant de plusieurs traits introduits à des moments différents de la genèse. Un trait, le parcours, peut être présent, sous une forme plus ou moins explicite, à toutes les étapes. La thématisation, introduite postérieurement, est aussi une partie intégrante du dispositif énonciatif des questions; elle est l'opération par laquelle l'énonciateur signifie que la variable doit être remplacée par une valeur; elle est responsable, en outre, de la syntaxe de surface courante des énoncés interrogatifs. D'autres traits pertinents peuvent s'ajouter; on doit noter que la thématisation, qui sélectionne et fixe une référence afin qu'elle reçoive un apport, est une opération 
générale qui entre dans la construction d'autres structures syntaxiques que celles étudiées.

\section{BIBLIOGRAPHIE}

Boone, A. \& Joly, A. (2004). Dictionnaire terminologique de la systématique du langage. Paris : L'Harmattan.

Cotte, P. (1997). L'explication grammaticale de textes anglais. Paris : Presses Universitaires de France.

Culioli, A. (1985). Notes du séminaire de DEA 1983-1984. Université de Poitiers.

Culioli, A. (1990). Pour une linguistique de l'énonciation. t. 1. Paris/Gap : Ophrys.

Culioli, A. (1999) Pour une linguistique de l'énonciation. t. 2-3. Paris/Gap : Ophrys.

Dixon, R. (2005). A Semantic Approach to English Grammar. Oxford : Oxford University Press.

Dufaye, L. (2006). « WH : fin de parcours ». In Gournay, L. \& Mélis, G. (éds) (2006). Revue Corela, volume hors-série $n^{\circ} 4:$ https://corela.revues.org/828

Gournay, L. (2005). « (Entre autres choses) pourquoi les marqueurs simples en WH ne sont finalement pas des opérateurs de parcours ». In Girard-Gillet, G. (éd.). Parcours linguistiques Domaine anglais. CIEREC Travaux 122, Université de Saint Etienne, 59-70.

Gournay, L. \& Mélis, G. (éds) (2006). Le Parcours. Revue Corela, volume hors-série nº 4 : https:// corela.revues.org/828

Groussier M.-L. \& Rivière C. (1996). Les mots de la linguistique, Lexique de linguistique énonciative. Paris : Ophrys, 1996

Guillaume, G. (1973). Leçons de linguistique de Gustave Guillaume 1948-1949. Québec : Les Presses de l'Université Laval, Paris : Librairie C. Klincksieck

Guillaume G. (1989). Leçons de linguistique de Gustave Guillaume 1946-1947c. Lille : Presses Universitaires de Lille, Québec : Presses de l'Université Laval.

Halliday, M. \& Matthiessen, C. (2004). An Introduction to Functional Grammar ( $3^{\text {rd }}$ ed.). London : Arnold.

Huddleston, R. \& Pullum, G. (2002). The Cambridge Grammar of the English Language.

Cambridge : Cambridge University Press.

Jespersen, O. (1924) The Philosophy of Grammar. London : Allen and Unwin.

Lalande, A. (1926). Vocabulaire technique et critique de la philosophie. Paris : Presses Universitaires de France

Le Goffic, P. (1994). «Indéfinis, interrogatifs, relatifs : parcours avec ou sans issue ». Faits de langues $4,31-40$.

Le Goffic, P. (2002). « Marqueurs d'interrogation/indéfinition/subordination : essai de vue d'ensemble ». Verbum XXIV (4), 315-340. 
Le Goffic, P. (2007). « Les mots qu- entre interrogation, indéfinition et subordination : quelques repères ». Lexique $18,13-46$.

Le Goffic, P. (2015). « Indéfinis et interrogatifs : le cas du français ». Langue Française, 187, 111-136

Lowe, R. (2007). Introduction à la psychomécanique du langage I. Québec : Presses de l'Université Laval.

Lyons, J. (1977). Semantics. vol 2, Cambridge : Cambridge University Press.

Mauroy, R. (2003). « Quel wh- dans les interrogatives, les relatives et les clivées ? ». In Celle, A. \& Gresset, S. (éds). La subordination en anglais. Une approche énonciative. Toulouse : Presses Universitaires du Mirail. 111-129.

Mauroy, R. (2006). «Stabilité et qualification dans les WH dits de parcours ». In Gournay, L. \& Mélis, G. (éds). Revue Corela, volume hors-série ${ }^{\circ} 4:$ https://corela.revues.org/828

Mélis, G. 2006 « Peut-on différencier l'opération du parcours ? ». In Gournay L. \& Mélis G. (éds). Revue Corela, volume hors-série $\mathrm{n}^{\circ} 4$ : https://corela.revues.org/828

Peirce, C. (1931-1935). Collected Papers 1-6, Cambridge, Mass. : Harvard University Press.

Plantin, C. (2016). Dictionnaire de l'argumentation. Lyon : ENS éditions.

Valin, R. (1981). Perspectives psychomécaniques sur la syntaxe. Québec : Presses de l'Université Laval.

\section{NOTES}

1. Parce que la relation évoquée est générale, il est distingué seulement entre "thème » et «propos ». L'avantage du premier sur son concurrent «topique » est d'être courant dans l'analyse littéraire des textes.

2. Dans What happened? What did you do ? le participant signifié par what est un procès.

3. Deux sortes de questions polémiques existent. 1) L'allocutaire tient pour vrai mais l'énonciateur tient pour faux (cas envisagé supra). 2) L'énonciateur tient pour vrai et il connaît l'identité de ce à propos de quoi il interroge; il interroge pour contraindre l'énonciateur à reconnaitre un fait ; ex : And who helped you then ? (proposition logique sous-jacente I helped you).

4. L'énonciateur peut aussi savoir, et donner à entendre qu'il sait, ce qu'est le référent tout en taisant, provisoirement, l'identité ; one, plutôt que some, signifie cette "indétermination pour l'allocutaire ».

5. Des questions sur la relation temporelle entre un procès et un moment donné existent ; ex : Did it begin during/before/after that crisis? (questions polaires distinctes sur trois valeurs de la relation temporelle), Did it begin during or after that crisis? (question alternative offrant un choix entre deux valeurs). De telles questions peuvent suivre une question en when moyennant l'ellipse de leur début ; ex : When did it begin? during that crisis? / during or after that crisis? L'analyse de when n'est pas contredite : conformément à l'instruction portée par le morphème le questionnement cherche le moment du procès et les questions de fin précisent cette recherche. Une réponse positive à during that crisis ? satisfait à la question en when; négative, elle incite à une autre recherche ; une réponse positive à after that crisis? situe bien le procès dans une partie du temps, quitte à ce que des précisions la complètent ; une réponse négative fait de même implicitement et une réponse à une question alternative a les mêmes effets quelle qu'elle soit.

6. Le quantifieur universel every réalise un parcours additif. 
7. La relégation de some au second plan est bien montrée en référence aux lieux par la paire somewhere (adverbe composé indéfini posant l'existence d'un lieu) -> where? (adverbe interrogatif invitant à un parcours de valeurs). Pour la logique la variable est «liée » dans la proposition en some et « libre » dans celle en wh.

8. C'est aussi la position de Mélis (2006) : «Une théorisation des échelonnements, ou phases, d'opération, une élaboration théorique qui consisterait à définir non pas "simplement " les opérations mais leurs séquences en termes à la fois d'intrication et de strates opérationnelles apparaît comme un élément nécessaire à la linguistique de l'énonciation ».

9. En (7a) les informations nouvelles contenues dans la relative précisent seulement une information donnée (more than natural) ; l'information nouvelle pertinente est dans le focus de la proposition en be.

\section{RÉSUMÉS}

Les concepts de thème et de thématisation sont généralement considérés comme centraux dans l'analyse sémantique et pragmatique des textes et des énoncés; toutefois ils ne figurent guère dans les études sur les questions. L'article défend l'idée qu'ils sont essentiels pour comprendre les interrogatives; il utilise ces concepts dans le cadre d'une analyse génétique des structures interrogatives.

While the concepts theme and thematization are generally recognized as essential components of a semantic/pragmatic analysis of sentences and texts, they are absent from most treatments of questions. This paper argues they are also essential to an understanding of questions ; it attempts to provide a genetic account of interrogative structures using these concepts.

\section{INDEX}

Mots-clés : Wh-, Aux, thème, thématisation, genèse

Keywords : Wh-, Aux, theme, thematization, genesis

\section{AUTEUR}

\section{PIERRE COTTE}

Université Paris-Sorbonne, CELISO EA 7332

pierre.cotte@paris-sorbonne.fr 